\title{
A Study of Quasi-parton Distribution Functions in the Diquark Spectator Model
}

\section{Leonard Gamberg}

Division of Science, Penn State Berks, Reading, PA 19610, USA

E-mail: lpg10epsu.edu

\section{Zhong-Bo Kang}

Theoretical Division, MS B283, Los Alamos National Laboratory, Los Alamos, NM 87545, USA

E-mail: zkang@lanl.gov

\section{Ivan Vitev*}

Theoretical Division, MS B283, Los Alamos National Laboratory, Los Alamos, NM 87545, USA

E-mail: ivitevalanl.gov

\section{Hongxi Xing}

Theoretical Division, MS B283, Los Alamos National Laboratory, Los Alamos, NM 87545, USA

E-mail: hxing@lanl.gov

To facilitate lattice QCD calculations of nucleon structute, a set of quasi-parton distributions were recently introduced. These quasi-PDFs were shown to reduce to standard PDFs when the nucleon is boosted to high energies, $P_{z} \rightarrow \infty$. Since taking such limit is not feasible in lattice simulations, it is essential to provide guidance for what values of $P_{z}$ the quasi-PDFs are good approximations of standard PDFs. Within the framework of the spectator diquark model, we evaluate both the up and down quarks' quasi-PDFs and standard PDFs for all leading-twist distributions (unpolarized distribution $f_{1}$, helicity distribution $g_{1}$, and transversity distribution $h_{1}$ ). We find that, for intermediate parton momentum fractions $x$, quasi-PDFs are good approximations to standard PDFs (within 20-30\%) when $P_{z} \gtrsim 1.5-2 \mathrm{GeV}$. On the other hand, for large $x \sim 1$ much larger $P_{z}>4 \mathrm{GeV}$ is necessary to obtain a satisfactory agreement between the two sets. We further find that the Soffer positivity bound does not hold in general for quasi-PDFs.

QCD Evolution 2015

May 26-30, 2015

Jefferson Lab (JLAB), Newport News Virginia, USA

\footnotetext{
* Speaker.
} 


\section{Introduction}

In recent years, evaluation of parton distribution functions (PDFs), fundamental non-perturbative ingredients of the QCD factorizaton approach, has been attempted in lattice QCD [1, 2, 3, 4]. Since PDFs are defined as the non-local light-cone correlations which involve the real Minkowski time, the traditional lattice QCD approach does not allow one to compute the PDFs directly [5]; one can only calculate the lower moments of the PDFs, which are matrix elements of local operators [1,2]. Recently, new methods have been proposed $[5,6]$ to evaluate PDFs on the lattice in terms of socalled quasi-PDFs, which are defined as matrix elements of equal-time spatial correlators. These quasi-PDFs can be computed directly on the lattice $[7,8,9]$ and should reduce to the standard PDFs when the proton's momentum $P_{z} \rightarrow \infty$. While in practice the proton momentum on the lattice can never become infinite, one can only hopefully access finite but large enough momenta on the lattice to carry out relevant QCD simulations. Here, we present guidance regarding the magnitude of the proton momentum based upon the comparision of PDFs and quasi-PDFs in the spectator diquark model [10].

\section{Overview and definitions of standard PDFs and quasi-PDFs:}

We consider a nucleon of mass $M$ moving in the $z$-direction, with the momentum $P^{\mu}$ given by

$$
P^{\mu}=\left(P_{0}, 0_{\perp}, P_{z}\right) \equiv\left[P^{+}, P^{-}, 0_{\perp}\right] .
$$

Here and throughout the paper we use $\left(v_{0}, v_{\perp}, v_{z}\right)$ and $\left[v^{+}, v^{-}, v_{\perp}\right]$ to represent Minkowski and lightcone components for any four-vector $v^{\mu}$ respectively, with light-cone variables $v^{ \pm}=\left(v_{0} \pm v_{z}\right) / \sqrt{2}$. We thus have

$$
P^{-}=\frac{M^{2}}{2 P^{+}}, \quad P_{0}=\sqrt{P_{z}^{2}+M^{2}} \equiv P_{z} \delta, \quad \text { with } \quad \delta=\sqrt{1+\frac{M^{2}}{P_{z}^{2}}} .
$$

For the helicity distribution $g_{1}$ and the transversity distribution $h_{1}$ we also have to consider the nucleon with either longitudinal or transverse polarization. For pure longitudinal polarization, the polarization vector $S_{L}^{\mu}$ is given by

$$
S_{L}^{\mu}=\frac{1}{M}\left(P_{z}, 0_{\perp}, P_{0}\right) \equiv \frac{1}{M}\left[P^{+},-P^{-}, 0_{\perp}\right] .
$$

On the other hand, for pure transverse polarization, we have the polarization vector $S_{T}^{\mu}$

$$
S_{T}^{\mu}=\left(0, \vec{S}_{\perp}, 0\right) \equiv\left[0^{+}, 0^{-}, \vec{S}_{\perp}\right] .
$$

The polarization vectors satisfy the conditions $P \cdot S_{L}=P \cdot S_{T}=0$, and $S_{L}^{2}=-1$ and $S_{T}^{2}=-\vec{S}_{\perp}^{2}=-1$.

The three leading-twist standard collinear PDFs are defined on the light-cone with the following operator expressions [11]

$$
\begin{aligned}
& f_{1}(x)=\int \frac{d \xi^{-}}{4 \pi} e^{-i \xi^{-} k^{+}}\left\langle P\left|\bar{\psi}\left(\xi^{-}\right) \gamma^{+} U_{n}\left[\xi^{-}, 0\right] \psi(0)\right| P\right\rangle, \\
& g_{1}(x)=\int \frac{d \xi^{-}}{4 \pi} e^{-i \xi^{-} k^{+}}\left\langle P S\left|\bar{\psi}\left(\xi^{-}\right) \gamma^{+} \gamma_{5} U_{n}\left[\xi^{-}, 0\right] \psi(0)\right| P S\right\rangle, \\
& h_{1}(x)=\int \frac{d \xi^{-}}{4 \pi} e^{-i \xi^{-} k^{+}}\left\langle P S\left|\bar{\psi}\left(\xi^{-}\right) \gamma^{+} \gamma_{5} \gamma \cdot S_{T} U_{n}\left[\xi^{-}, 0\right] \psi(0)\right| P S\right\rangle,
\end{aligned}
$$


with $x=k^{+} / P^{+}$. We define the light-cone vector $n^{\mu}=\left[0^{+}, 1^{-}, 0_{\perp}\right]$ with $n^{2}=0$ and $n \cdot v=v^{+}$for any four-vector $v^{\mu}$, and the gauge link $U_{n}\left[\xi^{-}, 0\right]$ along the light-cone direction specified by $n$ is given by

$$
U_{n}\left[\xi^{-}, 0\right]=\exp \left(-i g \int_{0}^{\xi^{-}} d \eta^{-} A^{+}\left(\eta^{-}\right)\right) .
$$

On the other hand, the quasi-PDFs introduced by Ji [5] are equal-time spatial correlations along the $z$-direction, and have the following operator definitions

$$
\begin{aligned}
& \tilde{f}_{1}\left(x, P_{z}\right)=\int \frac{d \xi_{z}}{4 \pi} e^{-i \xi_{z} k_{z}}\left\langle P\left|\bar{\psi}\left(\xi_{z}\right) \gamma_{z} U_{n_{z}}\left[\xi_{z}, 0\right] \psi(0)\right| P\right\rangle, \\
& \tilde{g}_{1}\left(x, P_{z}\right)=\int \frac{d \xi_{z}}{4 \pi} e^{-i \xi_{z} k_{z}}\left\langle P S\left|\bar{\psi}\left(\xi_{z}\right) \gamma_{z} \gamma_{5} U_{n_{z}}\left[\xi_{z}, 0\right] \psi(0)\right| P S\right\rangle, \\
& \tilde{h}_{1}\left(x, P_{z}\right)=\int \frac{d \xi_{z}}{4 \pi} e^{-i \xi_{z} k_{z}}\left\langle P S\left|\bar{\psi}\left(\xi_{z}\right) \gamma_{z} \gamma_{5} \gamma \cdot S_{T} U_{n_{z}}\left[\xi_{z}, 0\right] \psi(0)\right| P S\right\rangle,
\end{aligned}
$$

where $n_{z}^{\mu}=\left(0,0_{\perp}, 1\right)$ with $n_{z}^{2}=-1$ and $n_{z} \cdot v=-v_{z}$ for any four-vector $v^{\mu}$, where now the gauge link $U_{n_{z}}\left[\xi_{z}, 0\right]$ is along the direction of $n_{z}$ and is given by

$$
U_{n_{z}}\left[\xi_{z}, 0\right]=\exp \left(-i g \int_{0}^{\xi_{z}} d \eta_{z} A_{z}\left(\eta_{z}\right)\right)
$$

\section{The spectator diquark model}

The spectator diquark model of the nucleon has been described in great detail $[12,13,14,15]$. Here, we present a brief overview. In the spectator diquark model, the PDFs which are traces of the quark-quark correlation functions as defined in the last section are evaluated in the spectator approximation. In this framework a sum over a complete set of intermediate on-shell states, $I=$ $\sum_{X}|X\rangle\langle X|$, is inserted into the operator definition of PDFs, and truncated to single on-shell diquark spectator states with $X$ being either spin 0 (scalar diquark) or spin 1 (axial-vector diquark). The quark-quark correlation function is then obtained as the cut tree level amplitude for nucleon $N \rightarrow$ $q+X$ where $X=\{s, a\}$. With such an approximation, the nucleon is composed of a constituent quark of mass $m$ and a spectator scalar (axial-vector) diquark with mass $M_{s}\left(M_{a}\right)$. The interaction

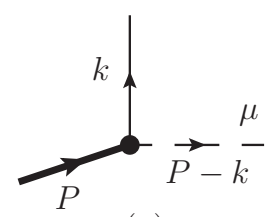

(a)

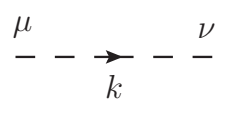

(b)

Figure 1: Feynman rules in the spectator diquark model: (a) vertex representing the interaction between the quark, the nucleon, and the diquark, (b) the diquark propagator.

between the nucleon, the quark, and the diquark is given by the following Feynman rules for the vertex in Fig. 1(a),

scalar diquark: $\quad i g_{s} \mathscr{I}_{s}\left(k^{2}\right), \quad$ axial-vector diquark: $\quad i \frac{g_{a}}{\sqrt{2}} \gamma^{\mu} \gamma_{5} \mathscr{I}_{a}\left(k^{2}\right)$, 
where following $[13,14,15]$, we have introduced suitable form factors $\mathscr{I}_{s, a}\left(k^{2}\right)$ as a function of $k^{2}$ - the invariant mass of the constituent quark. For our numerical calculations below, we adopt the fitted parameters in [14] and use the dipolar form factors,

$$
\mathscr{I}_{s}\left(k^{2}\right)=\frac{k^{2}-m^{2}}{\left(k^{2}-\Lambda_{s}^{2}\right)^{2}}, \quad \mathscr{I}_{a}\left(k^{2}\right)=\frac{k^{2}-m^{2}}{\left(k^{2}-\Lambda_{a}^{2}\right)^{2}},
$$

where $\Lambda_{s, a}$ are the appropriate cutoffs, to be considered as free parameters of the model together with the diquark masses $M_{s, a}$, and the couplings $g_{s, a}$. Further, the propagators of the scalar diquark and the axial-vector diquark as shown in Fig. 1(b) are given by the expressions,

$$
\text { scalar diquark: } \frac{i}{k^{2}-M_{s}^{2}}, \quad \text { axial-vector diquark: } \frac{i}{k^{2}-M_{a}^{2}} d^{\mu v}(k, n),
$$

where for the standard light-cone PDFs with $n^{2}=0$, we have [14, 15]

$$
d^{\mu v}(k, n)=-g^{\mu v}+\frac{n^{\mu} k^{v}+n^{v} k^{\mu}}{n \cdot k}-\frac{k^{2} n^{\mu} n^{v}}{(n \cdot k)^{2}},
$$

which satisfies $n_{\mu} d^{\mu v}(k, n)=k_{\mu} d^{\mu v}(k, n)=0$. On the other hand, for the quasi-PDFs, since $n_{z}^{2}=$ $-1 \neq 0$, we have a slightly different form for the polarization tensor $d^{\mu v}$ as

$$
d^{\mu v}\left(k, n_{z}\right)=-g^{\mu v}+\frac{n_{z} \cdot k}{\left(n_{z} \cdot k\right)^{2}-n_{z}^{2} k^{2}}\left(n_{z}^{\mu} k^{v}+n_{z}^{v} k^{\mu}\right)-\frac{1}{\left(n_{z} \cdot k\right)^{2}-n_{z}^{2} k^{2}}\left(k^{2} n_{z}^{\mu} n_{z}^{v}+n_{z}^{2} k^{\mu} k^{v}\right)
$$

which also satisfies $n_{z \mu} d^{\mu v}\left(k, n_{z}\right)=k_{\mu} d^{\mu v}\left(k, n_{z}\right)=0$.

\section{Standard PDFs and Quasi-PDFs in the spectator diquark model}

We give one detailed example of the calculation of standard PDFs and quasi-PDFs. In the scalar diquark model [12], $f_{1}^{s}\left(x, k_{\perp}^{2}\right)$ is given by

$$
\begin{aligned}
f_{1}^{s}\left(x, k_{\perp}^{2}\right)= & g_{s}^{2} \int \frac{d k^{+} d k^{-}}{(2 \pi)^{4}} \frac{1}{2 P^{+}} \delta\left(x-\frac{k^{+}}{P^{+}}\right) \operatorname{Tr}\left[\gamma \cdot n(\gamma \cdot k+m) \frac{1}{2}(\gamma \cdot P+M)(\gamma \cdot k+m)\right] \\
& \times \frac{1}{\left(k^{2}-m^{2}\right)^{2}} 2 \pi \delta\left((P-k)^{2}-M_{s}^{2}\right)\left[\mathscr{I}_{s}\left(k^{2}\right)\right]^{2},
\end{aligned}
$$

where the superscript " $s$ " in $f_{1}^{s}$ indicates that the diquark is a scalar, and $k_{\perp}$ is the quark transverse momentum [16, 17]. Eventually we obtain

$$
f_{1}^{s}\left(x, k_{\perp}^{2}=\frac{g_{s}^{2}}{(2 \pi)^{3}} \frac{(1-x)\left[k_{\perp}^{2}+(m+x M)^{2}\right]}{2\left[k_{\perp}^{2}+x M_{s}^{2}-x(1-x) M^{2}+(1-x) m^{2}\right]^{2}}\left[\mathscr{I}_{s}\left(k^{2}\right)\right]^{2},\right.
$$

where the invariant mass $k^{2}=-\frac{1}{1-x}\left[k_{\perp}^{2}+x M_{s}^{2}-x(1-x) M^{2}\right]$.

Using the definition of the cut vertices for the quasi-PDFs, we write the quasi-PDF $\tilde{f}_{1}^{s}\left(x, k_{\perp}^{2}, P_{z}\right)$ for the scalar diquark case as

$$
\begin{aligned}
\tilde{f}_{1}^{s}\left(x, k_{\perp}^{2}, P_{z}\right)= & -g_{s}^{2} \int \frac{d k_{0} d k_{z}}{(2 \pi)^{4}} \frac{1}{2 P_{z}} \delta\left(x-\frac{k_{z}}{P_{z}}\right) \operatorname{Tr}\left[\gamma \cdot n_{z}(\gamma \cdot k+m) \frac{1}{2}(\gamma \cdot P+M)(\gamma \cdot k+m)\right] \\
& \times \frac{1}{\left(k^{2}-m^{2}\right)^{2}} 2 \pi \delta\left((P-k)^{2}-M_{s}^{2}\right)\left[\mathscr{I}_{s}\left(k^{2}\right)\right]^{2}
\end{aligned}
$$


where we have used $\gamma_{z}=-\gamma \cdot n_{z}$. After some algebraic manipulation, the corresponding quasi-PDF $\tilde{f}_{1}^{s}\left(x, k_{\perp}^{2}, P_{z}\right)$ is given by

$$
\tilde{f}_{1}^{s}\left(x, k_{\perp}^{2}, P_{z}\right)=\frac{g_{s}^{2}}{(2 \pi)^{3}} \frac{(2 x-1) M^{2}+2 x M m-M_{s}^{2}+m^{2}-2(1-x)^{2}\left(1-\rho_{s} \delta\right) P_{z}^{2}}{2 \rho_{s}(1-x)\left[2(1-x)\left(1-\rho_{s} \delta\right) P_{z}^{2}+M^{2}+M_{s}^{2}-m^{2}\right]^{2}}\left[\mathscr{I}_{s}\left(k^{2}\right)\right]^{2},
$$

where for the quasi-PDFs $k^{2}=2(1-x)\left(1-\rho_{s} \delta\right) P_{z}^{2}+M^{2}+M_{s}^{2}$.

We now study what happens to the quasi-PDF $\tilde{f}_{1}^{s}\left(x, k_{\perp}^{2}, P_{z}\right)$ in the limit of $P_{z} \rightarrow \infty$. Approximating $\rho_{s}$ and $\delta$ to $\mathscr{O}\left(M^{2} / P_{z}^{2}\right)$

$$
\rho_{s} \approx 1+\frac{k_{\perp}^{2}+M_{s}^{2}}{2(1-x)^{2} P_{z}^{2}}, \quad \delta \approx 1+\frac{M^{2}}{2 P_{z}^{2}}, \quad \text { and } \quad\left(1-\rho_{s} \delta\right) P_{z}^{2} \approx-\frac{k_{\perp}^{2}+M_{s}^{2}}{2(1-x)^{2}}-\frac{M^{2}}{2} .
$$

Substituting this expression into the equations above we find that

$$
\tilde{f}_{1}^{s}\left(x, k_{\perp}^{2}, P_{z} \rightarrow \infty\right)=f_{1}^{s}\left(x, k_{\perp}^{2}\right) .
$$

Thus, the quasi-PDF reduces to the standard PDF $f_{1}^{s}\left(x, k_{\perp}^{2}\right)$ as $P_{z} \rightarrow \infty$ limit ${ }^{1}$. This simply verifies the leading order matching calculations carried out in $[5,6]$.

The approximation in Eq. (4.5) we are using above seems quite reasonable. However, it is important to emphasize that such an approximation only holds when $(1-x)^{2} \sim \mathscr{O}(1)$. When we are studying the quasi-PDFs in the very large $x \sim 1$ region, the large $P_{z}$ expansion used in Eq. (4.5) breaks down, in which case the quasi-PDFs can deviate substantially from the standard PDFs. Such a breakdown is directly related to the existence of the factor $(1-x)^{2} P_{z}^{2}$ in our calculation, which is traced back to the on-shell condition of the diquark. Since such an on-shell condition is fairly generic [18], we expect that it will be quite difficult for the quasi-PDFs to approach the standard PDFs in the large $x \sim 1$ region. In this case, one has to boost the proton to much larger $P_{z}$. We will further illustrate this point in our numerical studies in the next section.

With the dipolar form factor $\mathscr{I}_{s}\left(k^{2}\right)$ given in Eq. (3.2), one can further integrate $f_{1}^{s}\left(x, k_{\perp}^{2}\right)$ over $k_{\perp}^{2}$ to obtain the collinear distribution $f_{1}^{s}(x)$ as

$$
f_{1}^{S}(x)=\int d^{2} k_{\perp} f_{1}^{S}\left(x, k_{\perp}^{2}\right)=2 \pi \int_{0}^{\infty} d k_{\perp} k_{\perp} f_{1}^{S}\left(x, k_{\perp}^{2}\right),
$$

from which we obtain

$$
f_{1}^{s}(x)=\frac{g_{s}^{2}}{(2 \pi)^{2}} \frac{\left[2(m+x M)^{2}+L_{s}^{2}\left(\Lambda_{s}^{2}\right)\right](1-x)^{3}}{24 L_{s}^{6}\left(\Lambda_{s}^{2}\right)},
$$

with $L_{s}^{2}\left(\Lambda_{s}^{2}\right)$ defined as

$$
L_{s}^{2}\left(\Lambda_{s}^{2}\right) \equiv x M_{s}^{2}+(1-x) \Lambda_{s}^{2}-x(1-x) M^{2} .
$$

Now let us consider the quasi-PDF $\tilde{f}_{1}^{s}\left(x, P_{z}\right)$. We have

$$
\tilde{f}_{1}^{s}\left(x, P_{z}\right)=\int d^{2} k_{\perp} \tilde{f}_{1}^{s}\left(x, k_{\perp}^{2}, P_{z}\right)=2 \pi \int_{0}^{\infty} d k_{\perp} k_{\perp} \tilde{f}_{1}^{s}\left(x, k_{\perp}^{2}, P_{z}\right),
$$

\footnotetext{
${ }^{1}$ Though obvious, it is worthwhile emphasizing that this conclusion is independent of the fact whether one has the form factor $\mathscr{I}_{S}\left(k^{2}\right)$ in the spectator diquark model.
} 
with $\tilde{f}_{1}^{s}\left(x, k_{\perp}^{2}, P_{z}\right)$ given by Eq. (4.4). Because of the complicated functional form for $\tilde{f}_{1}^{s}\left(x, k_{\perp}^{2}, P_{z}\right)$, we are not able to obtain a simple analytical expression for the collinear quasi-PDF $\tilde{f}_{1}^{s}\left(x, P_{z}\right)$ and will only present the numerical studies for the collinear quasi-PDFs in the next section. Here, it is important to emphasize that, since in the limit of $P_{z} \rightarrow \infty, \tilde{f}_{1}^{s}\left(x, k_{\perp}^{2}, P_{z}\right)$ reduces to $f_{1}^{s}\left(x, k_{\perp}^{2}\right)$ as we have shown above, the collinear counter-part $\tilde{f}_{1}^{s}\left(x, P_{z}\right)$ also reduces to the standard collinear PDF $f_{1}^{s}(x)$.

We proceed to calculate the unintegrated helicity and transversity distributions $g_{1}^{s}\left(x, k_{\perp}\right), g_{1}^{a}\left(x, k_{\perp}\right)$, $h_{1}^{s}\left(x, k_{\perp}\right), h_{1}^{a}\left(x, k_{\perp}\right)$ for scalar and axial diquarks. We also obtain the quasi-helicity and quasitransversity distributions $\tilde{g}_{1}^{s}\left(x, k_{\perp}, P_{z}\right), \tilde{g}_{1}^{a}\left(x, k_{\perp}, P_{z}\right), \tilde{h}_{1}^{s}\left(x, k_{\perp}, P_{z}\right), \tilde{h}_{1}^{a}\left(x, k_{\perp}, P_{z}\right)$. From the unintegrated distributions one can proceed to get the integrated ones and all details are given in Ref. [10].

\section{Phenomenological results}

Following Ref. [14], the $u$-quark and $d$-quark unpolarized PDFs $f_{1}^{u, d}$ can be written as

$$
f_{1}^{u}=c_{s}^{2} f_{1}^{u(s)}+c_{a}^{2} f_{1}^{u(a)}, \quad f_{1}^{d}=c_{a}^{\prime 2} f_{1}^{d\left(a^{\prime}\right)},
$$

that is, the $u$-quark receives contributions from both scalar and axial-vector diquark, while the $d$ quark only has the axial-vector diquark contribution. Here the superscript " $s$ " represents the scalar diquark contribution, " $a$ " corresponds to the axial-vector diquark which has isospin 0 (isoscalar $u d$ like system), and " $a$ " denotes the axial-vector diquark contribution which has isospin 1 (isovector $u u$-like system). Thus, we have the following 9 model parameters: $c_{s, a}, c_{a}^{\prime}, M_{s, a}, M_{a}^{\prime}, \Lambda_{s, a}$, and $\Lambda_{a}^{\prime}$, as well as three couplings $g_{s}, g_{a}$, and $g_{a}^{\prime}$. We use the same method specified in [14] to fix these three couplings:

$$
\pi \int_{0}^{1} d x \int_{0}^{\infty} d k_{\perp}^{2} f_{1}^{q(X)}\left(x, k_{\perp}^{2}\right)=1,
$$

with $X=s, a, a^{\prime}$. On the other hand, the other 9 model parameters are fixed through a global fitting of both $f_{1}^{u}(x), f_{1}^{d}(x)$ at factorization scale $\mu^{2}=0.30 \mathrm{GeV}^{2}$ with ZEUS2002 PDFs [19] and $g_{1}^{u}(x)$, $g_{1}^{d}(x)$ at $\mu^{2}=0.26 \mathrm{GeV}^{2}$ with GRSV2000 [20] at leading order in [14]; the fit is satisfactory and gives consistent shape and size of the standard PDFs. In the following, we simply use these fitted parameters in our numerical study.

In Fig. 2, we plot the quasi-unpolarized distribution $x \tilde{f}_{1}\left(x, P_{z}\right)$ as a function of momentum fraction $x$ for both up quark (left panel) and down quark (right panel) at different values of $P_{z}$; $1 \mathrm{GeV}$ (purple), $2 \mathrm{GeV}$ (green), $3 \mathrm{GeV}$ (blue), and $4 \mathrm{GeV}$ (red), respectively. For comparison, the standard unpolarized distribution $x f_{1}(x)$ is also shown (black dashed curve). It is important to realize that the quasi-PDFs have support for $-\infty<x<+\infty[5,6,18]$, and thus quasi-PDFs do not vanish for $x>1$ at finite $P_{z}$. This is clearly seen in the figures: while $f_{1}(x) \rightarrow 0$ as $x \rightarrow 1$ for both $u$ and $d$ quarks, at finite $P_{z}, \tilde{f}_{1}\left(x, P_{z}\right)$ remains finite when $x \rightarrow 1$. It is evident that $\tilde{f}_{1}\left(x, P_{z}\right)$ has different behavior as compared with the standard distribution $f_{1}(x)$ for relatively small $P_{z}=1 \mathrm{GeV}$, as shown by the purple curves in Fig. 2. However, once one increases $P_{z} \geq 2 \mathrm{GeV}$, the shape of the quasi-PDFs approaches those of the standard PDFs.

In Figs. 3 we plot the quasi-helicity distribution $x \tilde{g}_{1}\left(x, P_{z}\right)$. We find very similar features to the unpolarized case. For small $P_{z}=1 \mathrm{GeV}$, the quasi-PDFs are different from the standard PDFs, but again, increasing $P_{z} \geq 2 \mathrm{GeV}$, they become similar to the standard PDFs. Transversity distributions 

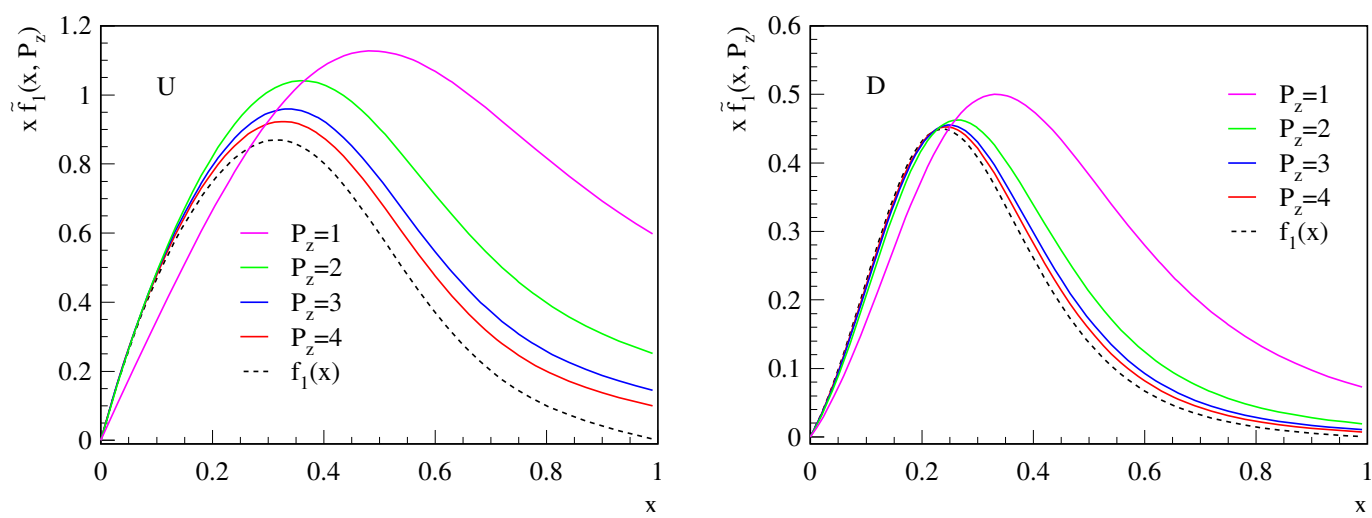

Figure 2: The unpolarized quasi-PDFs $x \tilde{f}_{1}\left(x, P_{z}\right)$ are plotted as a function of $x$ for $u$ (left) and $d$ (right) quark, respectively. Different lines are shown for $P_{z}=1 \mathrm{GeV}$ (purple), $2 \mathrm{GeV}$ (green), $3 \mathrm{GeV}$ (blue), and $4 \mathrm{GeV}$ (red), respectively. The standard PDF $f_{1}(x)$ (black dashed) is also shown for comparison.
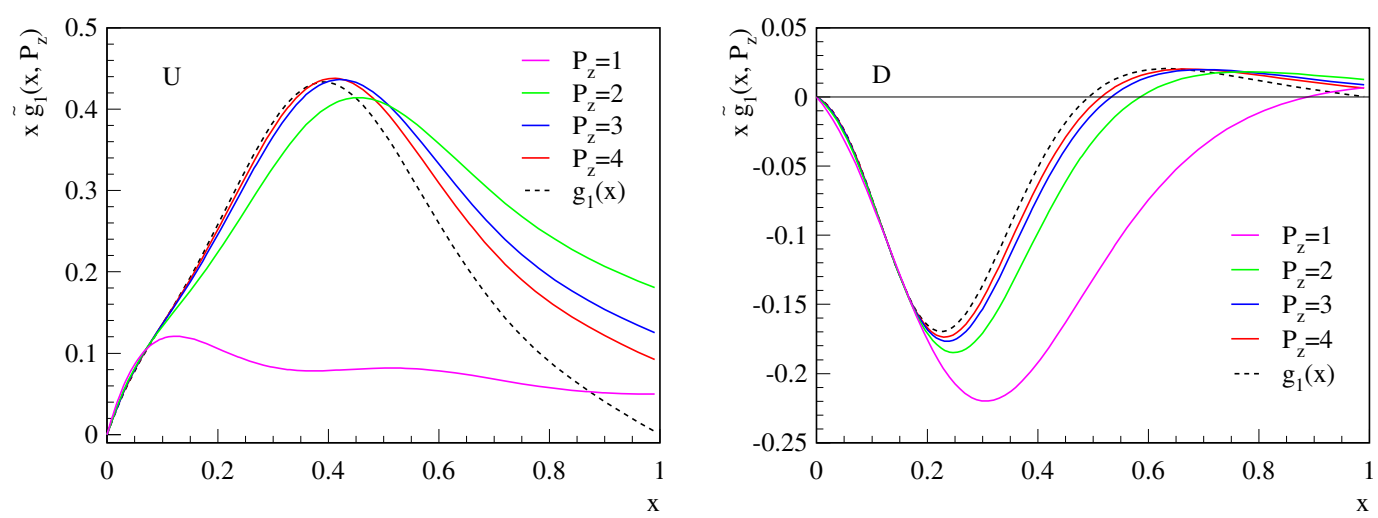

Figure 3: The helicity quasi-PDFs $x \tilde{g}_{1}\left(x, P_{z}\right)$ are plotted as a function of $x$ for $u$ (left) and $d$ (right) quark, respectively. Different lines are shown for $P_{z}=1 \mathrm{GeV}$ (purple), $2 \mathrm{GeV}$ (green), $3 \mathrm{GeV}$ (blue), and $4 \mathrm{GeV}$ (red), respectively. The standard helicity distribution $g_{1}(x)$ (black dashed) is also shown for comparison.

can be found in our paper [10]. To further study the relative difference between quasi-PDFs and standard PDFs quantitatively, we define the following ratios:

$$
R_{f}^{q}\left(x, P_{z}\right)=\frac{\tilde{f}_{1}^{q}\left(x, P_{z}\right)}{f_{1}^{q}(x)}, \quad R_{g}^{q}\left(x, P_{z}\right)=\frac{\tilde{g}_{1}^{q}\left(x, P_{z}\right)}{g_{1}^{q}(x)}, \quad R_{h}^{q}\left(x, P_{z}\right)=\frac{\tilde{h}_{1}^{q}\left(x, P_{z}\right)}{h_{1}^{q}(x)},
$$

As can be seen above for the intermediate $0.1 \lesssim x \lesssim 0.4-0.5$ all the quasi-PDFs approximate the corresponding standard PDFs to within $20-30 \%$ when $P_{z} \gtrsim 1.5-2 \mathrm{GeV}$, which seems within reach of lattice QCD calculations [7]. On the other hand, as we have emphasized in last section, for the very large $x \sim 1$ region, the quasi-PDFs could be quite different from standard PDFs. This has already been demonstrated in Figs. 2, 3, where the quasi-PDFs are still finite but the standard PDFs all vanish when $x \rightarrow 1$. Let us further make this point. In Fig. 4, we plot the ratio $R^{q}\left(x, P_{z}\right)$ at large $x=0.7$ as a function of $P_{z}$ for $f_{1}^{u}$ (red), $f_{1}^{d}$ (blue), $g_{1}^{u}$ (green), and $h_{1}^{d}$ (purple), respectively. One can see that at $P_{z} \sim 1-2 \mathrm{GeV}$, the ratio can be as large as $6-7$; that is, in the large $x$ kinematics regime, the quasi-PDFs are quite different from the standard PDFs. In this kinematic regime, one has to go to very large $P_{z}>4 \mathrm{GeV}$ at least to obtain a good approximation to the standard PDFs. 


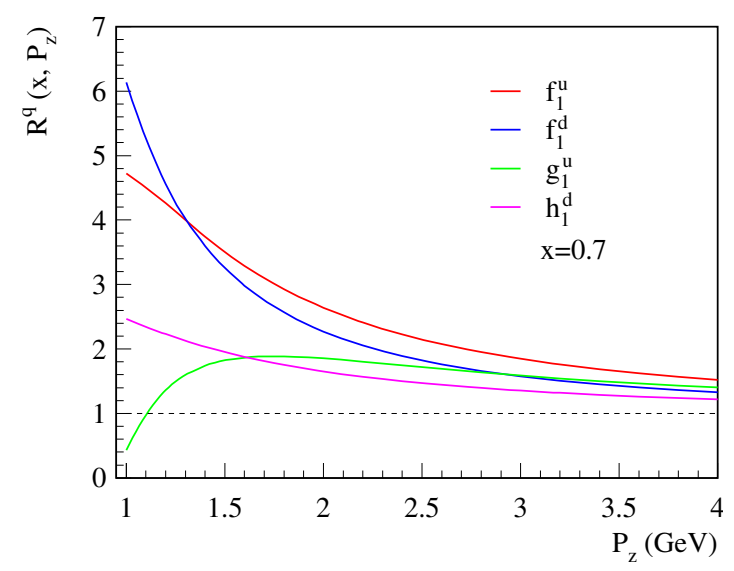

Figure 4: The ratio $R^{q}\left(x, P_{z}\right)$ at large $x=0.7$ as a function of $P_{z}$ for $f_{1}^{u}$ (red), $f_{1}^{d}$ (blue), $g_{1}^{u}$ (green), and $h_{1}^{d}$ (purple), respectively.

\subsection{Positivity bound: Soffer inequality}

The Soffer inequality [21] relates the three leading-twist collinear PDFs $f_{1}, g_{1}$, and $h_{1}$ as

$$
\left|h_{1}^{q}(x)\right| \leq \frac{1}{2}\left(f_{1}^{q}(x)+g_{1}^{q}(x)\right) .
$$

To test such an inequality for both standard PDFs and quasi-PDFs, let us define the following quantities:

$$
\begin{aligned}
\tilde{S}^{q}\left(x, P_{z}\right) & =\frac{1}{2}\left(f_{1}^{q}\left(x, P_{z}\right)+g_{1}^{q}\left(x, P_{z}\right)\right)-\left|h_{1}^{q}\left(x, P_{z}\right)\right|, \\
S^{q}(x) & =\frac{1}{2}\left(f_{1}^{q}(x)+g_{1}^{q}(x)\right)-\left|h_{1}^{q}(x)\right| .
\end{aligned}
$$

The Soffer bound holds for the standard PDFs, thus we have $S^{q}(x) \geq 0$.
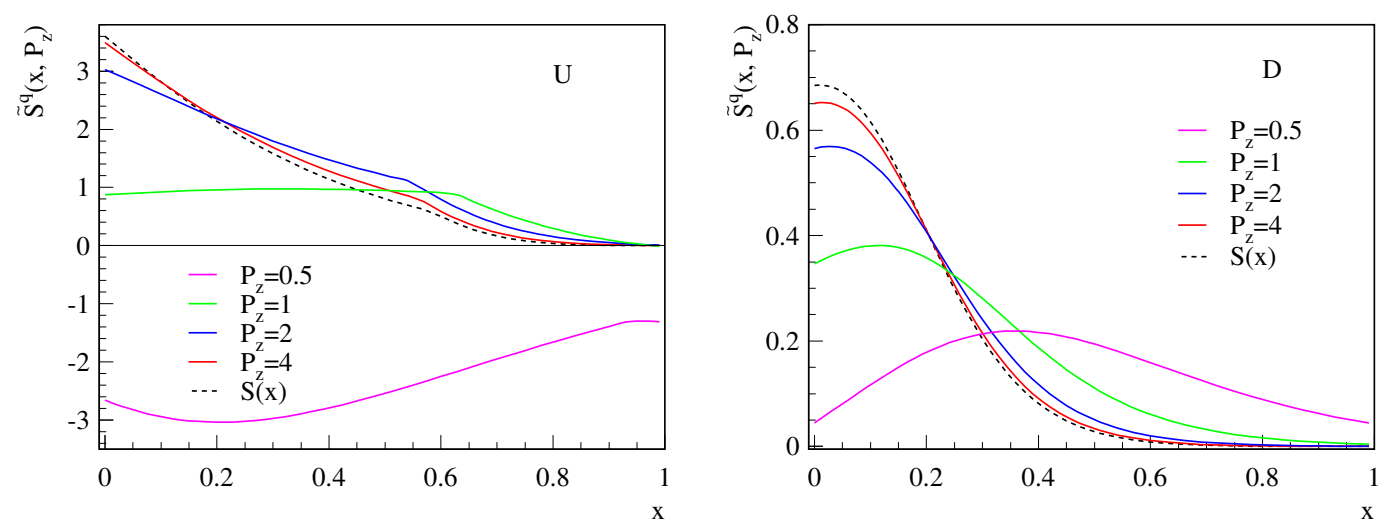

Figure 5: The function $\tilde{S}^{q}\left(x, P_{z}\right)$ is plotted versus $x$ for $u$ (left) and $d$ (right) quark. Different lines are shown for $P_{z}=0.5 \mathrm{GeV}$ (purple), $1 \mathrm{GeV}$ (green), $2 \mathrm{GeV}$ (blue), $4 \mathrm{GeV}$ (red), respectively. The function $S^{q}(x)$ (black dashed) is also shown for comparison.

In Fig. 5, $\tilde{S}^{q}\left(x, P_{z}\right)$ is plotted versus $x$ for $u$ (left) and $d$ (right) quark at different values of $P_{z}$, $0.5 \mathrm{GeV}$ (purple), $1 \mathrm{GeV}$ (green), $2 \mathrm{GeV}$ (blue), and $4 \mathrm{GeV}$ (red), respectively. The function $S^{q}(x)$ 
(black dashed) for the standard PDFs is also shown for comparison. As one can see clearly from the black dashed curves, the Soffer bound is indeed satisfied for the standard PDFs for both $u$ and $d$ quarks. At the same time, within our spectator diquark model, as shown in the right panel of Fig. 5, for all the selected $P_{z}$ values, $\tilde{S}^{q}\left(x, P_{z}\right) \geq 0$ for the $d$ quark, that is, the Soffer bound appears to be satisfied for the $d$-quark quasi-PDFs. On the other hand, as shown in the left panel of Fig. 5 for the $u$ quark, even though $\tilde{S}^{q}\left(x, P_{z}\right) \geq 0$ for $P_{z}=1,2$, and $4 \mathrm{GeV}$, for $P_{z}=0.5 \mathrm{GeV}, \tilde{S}^{q}\left(x, P_{z}\right)<0$ for the entire plotted $0 \leq x \leq 1$ region. In other words, the Soffer bound breaks down for relatively small $P_{z}$ values for the $u$ quark. What this tells us for the usual lattice QCD simulations is that while the standard PDFs might still satisfy the positivity bounds, such as Soffer bound on the lattice [22], these positivity bounds in general do not hold for quasi-PDFs, and, thus, one should avoid using them in lattice simulations.

\section{Conclusions}

We used the spectator diquark model to consistently compare the quasi-parton distribution functions (PDFs) and the standard PDFs. We took into account both the scalar diquark and axialvector diquark contributions and generated all the three leading-twist collinear PDFs, the unpolarized distribution $f_{1}$, the helicity distribution $g_{1}$, and the transversity distribution $h_{1}$. Using the model parameters which lead to a reasonable description of the standard PDFs $f_{1}^{u, d}(x)$ and $g_{1}^{u, d}(x)$, consistent with those extracted from the global analysis [14], we presented numerical studies for all quasi-PDFs. We found that for intermediate $0.1 \lesssim x \lesssim 0.4-0.5$, the quasi-PDFs are good approximations for the corresponding standard PDFs when the proton momentum $P_{z} \gtrsim 1.5-2 \mathrm{GeV}$. However, in the large $x \sim 1$ region, a much larger $P_{z}>4 \mathrm{GeV}$ is necessary to obtain a similar accuracy of the approximation. By studying the Soffer positivity bound we found that the positivity bounds do not hold in general for the quasi-PDFs. Our study provides useful guidance for the lattice QCD calculations regarding the proton boost and accuracy of the quasi-PDFs approximation.

\section{Acknowledgements}

This work is supported by the U.S. Department of Energy under Contract Nos. DE-FG0207ER41460 (L.G.) and DE-AC02-05CH11231 (Z.K., I.V. and H.X.), and in part by the LDRD program at LANL.

\section{References}

[1] M. Deka et al., Phys.Rev. D79, 094502 (2009), arXiv:0811.1779.

[2] C. Alexandrou, EPJ Web Conf. 73, 01013 (2014), arXiv:1404.5213.

[3] P. Hagler, Phys.Rept. 490, 49 (2010), arXiv:0912.5483.

[4] B. Musch, P. Hagler, M. Engelhardt, J. Negele, and A. Schafer, Phys.Rev. D85, 094510 (2012), arXiv:1111.4249.

[5] X. Ji, Phys.Rev.Lett. 110, 262002 (2013), arXiv:1305.1539.

[6] Y.-Q. Ma and J.-W. Qiu, (2014), arXiv:1404.6860. 
[7] H.-W. Lin, J.-W. Chen, S. D. Cohen, and X. Ji, (2014), arXiv:1402.1462.

[8] C. Alexandrou et al., PoS LATTICE2014, 135 (2014), arXiv:1411.0891.

[9] K.-F. Liu, Quark and Glue Components of the Proton Spin from Lattice Calculation, in 21st International Symposium on Spin Physics (SPIN 2014) Beijing, China, October 20-24, 2014, 2015, arXiv:1504.06601.

[10] L. Gamberg, Z.-B. Kang, I. Vitev, and H. Xing, Phys. Lett. B743, 112 (2015), arXiv:1412.3401.

[11] CTEQ Collaboration, R. Brock et al., Rev.Mod.Phys. 67, 157 (1995).

[12] R. Jakob, P. Mulders, and J. Rodrigues, Nucl.Phys. A626, 937 (1997), arXiv:hep-ph/9704335.

[13] L. P. Gamberg, G. R. Goldstein, and M. Schlegel, Phys.Rev. D77, 094016 (2008), arXiv:0708.0324.

[14] A. Bacchetta, F. Conti, and M. Radici, Phys.Rev. D78, 074010 (2008), arXiv:0807.0323.

[15] Z.-B. Kang, J.-W. Qiu, and H. Zhang, Phys.Rev. D81, 114030 (2010), arXiv:1004.4183.

[16] A. Bacchetta, U. D’Alesio, M. Diehl, and C. A. Miller, Phys.Rev. D70, 117504 (2004), arXiv:hep-ph/0410050.

[17] A. Bacchetta et al., JHEP 0702, 093 (2007), arXiv:hep-ph/0611265.

[18] X. Xiong, X. Ji, J.-H. Zhang, and Y. Zhao, Phys.Rev. D90, 014051 (2014), arXiv:1310.7471.

[19] ZEUS Collaboration, S. Chekanov et al., Phys.Rev. D67, 012007 (2003), arXiv:hep-ex/0208023.

[20] M. Gluck, E. Reya, M. Stratmann, and W. Vogelsang, Phys.Rev. D63, 094005 (2001), arXiv:hep-ph/0011215.

[21] J. Soffer, Phys. Rev. Lett. 74, 1292 (1995), hep-ph/9409254.

[22] QCDSF Collaboration, UKQCD Collaboration, M. Diehl et al., p. 173 (2005), arXiv:hep-ph/0511032. 